\title{
Management of Adults with Acute Oesophageal Soft Food Bolus and Foreign Body Obstructions at Two New Zealand District Health Boards
}

\author{
Robert Hackett ${ }^{1,2}$ \\ Anthony R Brownson ${ }^{3}$ \\ Jason Hill $\mathbb{D}^{4}$ \\ Zoe Raos ${ }^{5}$
}

'Department of Gastroenterology, Bay of Plenty District Health Board, Tauranga, New Zealand; ${ }^{2}$ Department of Medicine, University of Otago, Wellington, New Zealand; ${ }^{3}$ Department of Medicine, Southern District Health Board, Dunedin, New Zealand; ${ }^{4}$ Department of Gastroenterology, Southern District Health Board, Dunedin, New Zealand; ${ }^{5}$ Department of Gastroenterology, Waitemata District Health Board, Auckland, New Zealand
Correspondence: Robert Hackett Tel +6475798000

Email rob_hackett@hotmail.co.uk

\begin{abstract}
Aim: 1. Investigate the characteristics of adult patients presenting with acute oesophageal soft food bolus obstruction (SFBO) and impacted foreign body (IFB) at two New Zealand district health boards (DHBs). 2. Review current management against international guidelines for SFBO and IFB.
\end{abstract}

Methods: A multicentre retrospective search of the Provation ${ }^{\circledR}$ endoscopy database identified patients presenting with acute oesophageal obstruction. Utilising electronic patient records, key data points including patient demographics, risk factors, pre-endoscopic medical therapies utilised, diagnostic radiological investigations performed and endoscopic complications were identified. Key timepoints and delays in the patient's hospital journey from oesophageal obstruction to therapeutic endoscopy were recorded. The probability of failing to undergo therapeutic endoscopy for SFBO within the timeframes advised in clinical guidelines as a result of a delay in referral to the endoscopy service was calculated.

Results: Over a cumulative 10.5-year period of data collection, 227 oesophago-gastroduodenoscopies were performed: 195 SFBO, 16 IFB, 16 no obstruction identified. Median patient age was 57 (15-95) years. 143 male and 84 female patients. Radiographs were performed in $50.9 \%$ of uncomplicated SFBO. Pre-endoscopy medical therapies were administered in $41.4 \%$ of the cases. Median time delay from onset of obstruction to therapeutic endoscopy varied: SFBO 19h 0min, complete obstruction $17 \mathrm{~h} 45 \mathrm{~min}$, impacted batteries $1 \mathrm{~h} 15 \mathrm{~min}$, and presumed sharp objects $6 \mathrm{~h} 0 \mathrm{~min}$. Three patients presenting with a soft food bolus obstruction failed to undergo therapeutic endoscopy due to a delay in referral to the endoscopy service, probability 0.034 (95\% CI 0.012, 0.095). Two patients died of complications secondary to oesophageal obstruction.

Discussion: Oesophageal obstruction is a common gastroenterological presentation. At two large centres in New Zealand, patients waited considerably longer than the recommended timeframe from obstruction to therapeutic endoscopy. Contributing factors included patientrelated delays to presentation, hospital system-related factors and delays in referral for endoscopy contributed to by unnecessary pre-endoscopic medical therapies and radiographic investigations. Education about oesophageal obstruction together with robust local guidelines have potential to reduce delays and length of hospital stay, as well as reduce patient discomfort and complications.

Keywords: oesophagus, obstruction, impaction, soft food bolus, foreign body

\section{Aim}

1. Investigate the characteristics of adult patients presenting with acute oesophageal soft food bolus obstruction (SFBO) and impacted foreign body (IFB) at two New Zealand district health boards (DHB). 
2. Review current management against international guidelines for SFBO and IFB, including pre-endoscopic investigations and management, and time to therapeutic endoscopy.

\section{Method}

Medline literature searches identified international clinical guidelines $^{1,2}$ on the management of acute oesophageal obstruction. A further search identified studies investigating the utility and efficacy of radiological investigations and medical therapies in the management of acute oesophageal obstruction.

The authors collaborated to identify key auditable standards against which current practice could be assessed (Table 1).

A retrospective search utilising the Provation ${ }^{\circledR}$ endoscopy database identified patients who underwent oesophago-gastro-duodenoscopy (OGD) for oesophageal obstruction, secondary to soft food bolus or ingested foreign body, between $1 / 6 / 10$ and $1 / 12 / 16$ at Waitemata DHB (6.5 years). A clinical care checklist pathway was created and implemented at Waitemata DHB in 2017 and made available nationally as a resource via the New Zealand
Society of Gastroenterology website in $2019 .{ }^{3}$ Colleagues at Southern DHB then replicated this retrospective study collecting data from $1 / 1 / 16$ to $31 / 12 / 19$ (4 years) and all data was combined.

Once cases were identified we utilised the electronic patient record to collect key data points, identified from our literature review (Appendix 1), which were recorded in an anonymised excel data collection spread sheet.

When the time of onset of obstruction was documented as "breakfast", "lunch" or "dinner" in the patient record, the times of 8:00h, 13:00h and 18:00h respectively were allocated as time of obstruction. The delay between each time point was calculated, along with the overall time delay from onset of oesophageal obstruction to therapeutic endoscopy (Figure 1). The time to therapeutic endoscopy was audited against those key auditable standards recommended in the international guidelines. Where available time of presentation was recorded to identify whether the time delays, utilisation of radiological investigation or medical therapies differed for patients who presented between 08:00 and 20:00 (Daytime) compared to 20:00 and 08:00 (Overnight).

For patients presenting with SFBO, with and without signs of complete oesophageal obstruction, where all key

Table I Key Auditable Outcomes Chosen from Published International Guidelines

\begin{tabular}{|c|c|c|}
\hline & $\begin{array}{l}\text { 2016: Removal of Foreign Bodies in the Upper } \\
\text { Gastrointestinal Tract in Adults: ESGE Guideline' }\end{array}$ & $\begin{array}{l}\text { 20 I I: Management of Ingested Foreign Bodies and } \\
\text { Food Impactions. ASGE Guideline }{ }^{2}\end{array}$ \\
\hline $\begin{array}{l}\text { Radiographs for } \\
\text { non-bony food } \\
\text { bolus obstructions }\end{array}$ & $\begin{array}{l}\text { ESGE does not recommend radiological evaluation for } \\
\text { patients with non-bony food bolus impaction without } \\
\text { complications }\end{array}$ & $\begin{array}{l}\text { For patients with suspected non-bony food bolus impaction } \\
\text { without complications (eg, no evidence of perforation, no } \\
\text { respiratory distress), endoscopy may be performed without } \\
\text { obtaining radiographs. }\end{array}$ \\
\hline Medical treatment & $\begin{array}{l}\text { The effectiveness of medical treatment of esophageal food } \\
\text { bolus impaction is debated. It is therefore recommended, } \\
\text { that medical treatment should not delay endoscopy }\end{array}$ & $\begin{array}{l}\text { Glucagon is relatively safe and thus remains an acceptable } \\
\text { option. Its use, however, should not delay definitive } \\
\text { endoscopic removal of a food impaction. }\end{array}$ \\
\hline $\begin{array}{l}\text { Emergent } \\
\text { endoscopy }\end{array}$ & $\begin{array}{l}\text { ESGE recommends emergent (preferably within } 2 \text { hours, but } \\
\text { at the latest within } 6 \text { hours) therapeutic } \\
\text { esophagogastroduodenoscopy for foreign bodies inducing } \\
\text { complete esophageal obstruction, and for sharp-pointed } \\
\text { objects or batteries in the esophagus. }\end{array}$ & $\begin{array}{l}\text { Emergent endoscopy: Patients with esophageal obstruction } \\
\text { (ie, unable to manage secretions), disk batteries, sharp- } \\
\text { pointed objects. }\end{array}$ \\
\hline Urgent endoscopy & $\begin{array}{l}\text { We recommend urgent (within } 24 \text { hours) therapeutic } \\
\text { esophagogastroduodenoscopy for other esophageal foreign } \\
\text { bodies without complete obstruction }\end{array}$ & $\begin{array}{l}\text { Esophageal foreign objects and food impactions should be } \\
\text { removed within } 24 \text { hours because delay decreases the } \\
\text { likelihood of successful removal and increases the risk of } \\
\text { complications including risk of perforation }\end{array}$ \\
\hline $\begin{array}{l}\text { Diagnostic work-up } \\
\text { for food bolus }\end{array}$ & $\begin{array}{l}\text { In cases of food bolus impaction, ESGE recommends } \\
\text { a diagnostic work-up for potential underlying disease, } \\
\text { including histological evaluation, in addition to therapeutic } \\
\text { endoscopy }\end{array}$ & \\
\hline
\end{tabular}




\begin{tabular}{|c|c|c|c|c|c|c|}
\hline $\begin{array}{c}\text { Onset of } \\
\text { oesophageal } \\
\text { obstruction }\end{array}$ & $\begin{array}{c}\text { Delay in } \\
\text { presentation } \\
\text { to hospital }\end{array}$ & $\begin{array}{c}\text { Arrival, } \\
\text { assessment } \\
\text { +/- initial } \\
\text { management } \\
\text { in hospital }\end{array}$ & $\begin{array}{c}\text { Referral to } \\
\text { endoscopy } \\
\text { service }\end{array}$ & $\begin{array}{l}\text { Assessment } \\
\text { by medical } \\
\text { team / } \\
\text { endoscopy } \\
\text { service }\end{array}$ & $\begin{array}{c}\text { Inpatient } \\
\text { wait }\end{array}$ & $\begin{array}{l}\text { Therapeutic } \\
\text { endoscopy }\end{array}$ \\
\hline \multicolumn{7}{|c|}{$\leftarrow$ Overall time delay oesophageal obstruction to therapeutic endoscopy $\rightarrow$} \\
\hline \multirow{3}{*}{\multicolumn{2}{|c|}{ Patient related delays }} & \multicolumn{5}{|c|}{$\leftarrow$ Door to Endoscopy time $\rightarrow$} \\
\hline & & \multicolumn{5}{|c|}{ Hospital systems related delays } \\
\hline & & \multicolumn{2}{|c|}{$\begin{array}{l}\text { Delay in referral to } \\
\text { endoscopy service }\end{array}$} & \multicolumn{3}{|c|}{$\begin{array}{l}\text { Inpatient delay to therapeutic } \\
\text { endoscopy }\end{array}$} \\
\hline
\end{tabular}

Figure I Key time points in patient journey and nomenclature of delays.

time points in the patients' journey had been documented (88 cases) we utilised the $\mathrm{R}$ Software for Statistical Computing to calculate the median time delay and $95 \%$ confidence interval, from hospital presentation to referral to the endoscopy service along with the probability of failing to undergo therapeutic endoscopy within the timeframe advised in clinical guidelines as a result of this delay.

\section{Ethics}

This anonymised retrospective study, carried out in accordance with the ethical principles set out in the declaration of Helsinki, was exempt from ethics review as per the Health and Disability Ethics Committee Standard Operating Procedure. As per the standard pre-procedural informed consent process, patients provided written consent for the use of endoscopic images to be used for research, education and teaching purposes.

\section{Results}

Over the period of data collection, 227 OGDs were performed in 203 patients for oesophageal obstruction. One hundred and forty-eight cases were managed at Waitemata DHB and 79 at Southern DHB. In total, 195 cases of soft food bolus obstruction (SFBO) and 16 cases of impacted foreign body (IFB) were endoscopically confirmed and managed. In 16 cases, the reported SFBO (9 cases) or IFB ( 7 cases) were not identified in the oesophagus, stomach or duodenum. In one case, the foreign body (toothbrush head) was located in the trachea. Multiple oesophageal obstructions were encountered in 19 patients with 15 patients experiencing two obstructions, three patients experiencing three obstructions and one patient experiencing four obstructions.
The median patient age was 57 years (range 15-95) with 143 male and 84 female patients. The majority of impactions $(91.6 \%)$ occurred in the patient's own private residence with $3.5 \%$ presenting from a rest home, $0.9 \%$ from a correctional facility, $0.5 \%$ from an inpatient psychiatric unit and $0.5 \%$ inpatient hospital ward. In seven cases $(3 \%)$ the usual place of residence was not documented.

Prior known risk factors for oesophageal obstruction were identified in 86/227 (38\%) with 27/227 (12\%) having two or more risk factors. The most common risk factor was a previous oesophageal obstruction, 65/227 (28.6\%), followed by eosinophilic oesophagitis and psychiatric disorder both 14/227 (6.1\%), oesophageal motility disorder 12/227 (5.2\%), Schatzki ring 10/227 (4.4\%), cognitive impairment or learning difficulty $8 / 227$ (3.5\%), oesophageal malignancy and oesophageal stricture both 5/227 (2.2\%), alcohol intoxication and history of gastric bypass both $3 / 227(1.3 \%)$ and taking a regular medication associated with the development of oesophagitis 2/227 (0.8\%) (Figure 2, Figure 3, Figure 4, Figure 5).

In patients who had suffered a prior obstruction (65 cases), an underlying pathology was identified in 23/65 (35\%); eosinophilic oesophagitis $9 / 23$ (39\%) and Schatzki ring $7 / 23$ (30\%) being the most common.

The type of soft food bolus was documented in 115 of 204 cases (Figures 3-5). The majority, 101/115 (87.8\%), were meat products with beef steak $43 \%$, chicken $18 \%$ and lamb $13 \%$ being the most common. Of the 16 cases of foreign body ingestion, 12 cases were sharp objects (of which two also ingested batteries) and four were blunt objects. Sharp objects included razor blades $4 / 12$, beer bottle cap $3 / 12$, pin $2 / 12$, pencil $1 / 12$, disassembled watch $1 / 12$ and sharp piece of lamb 


\section{Established risk factors in 227 patients presenting with signs or symptoms of oesophageal obstruction}

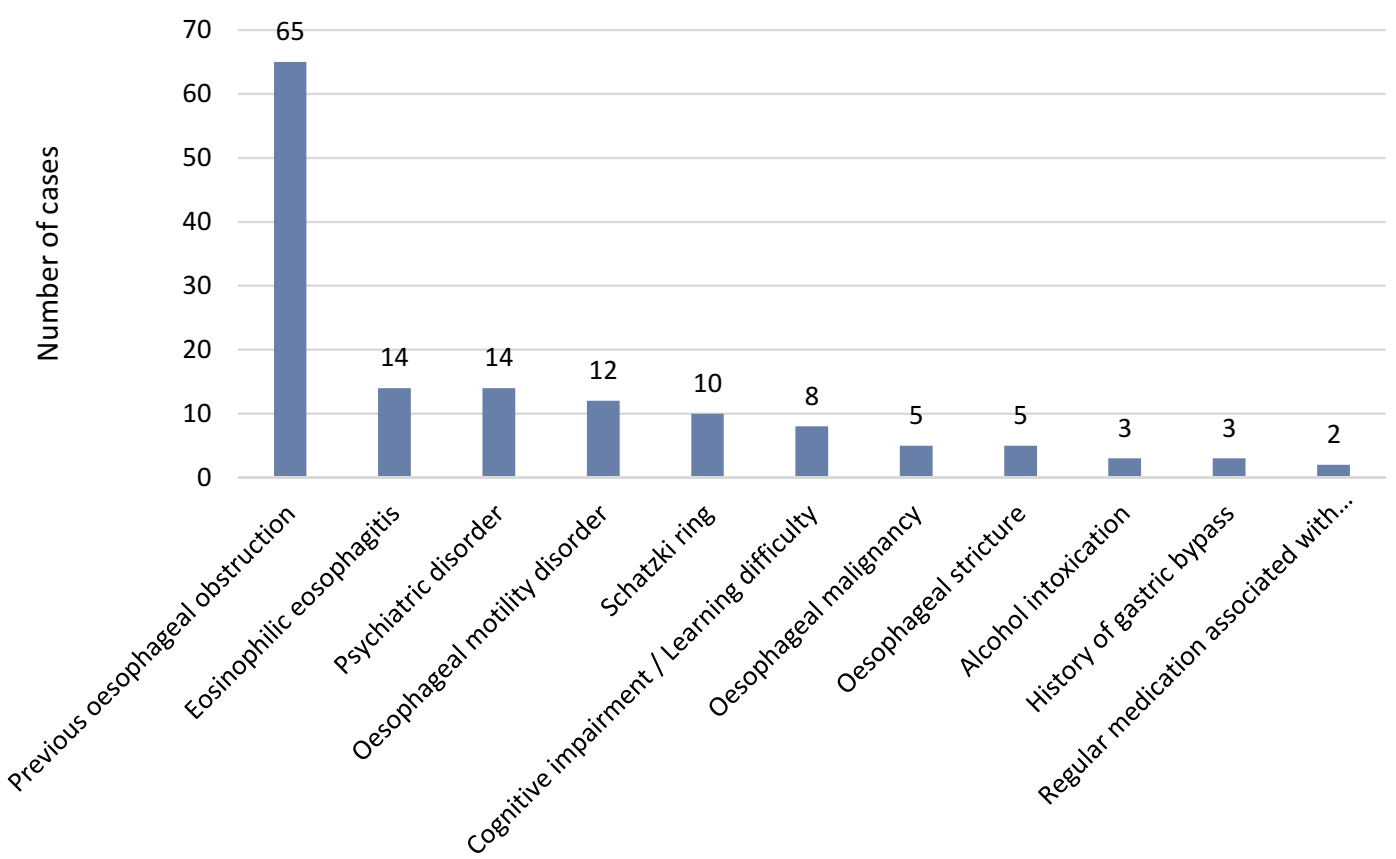

Risk factor

Figure 2 Established risk factors in 227 patients presenting with signs or symptoms of oesophageal obstruction.

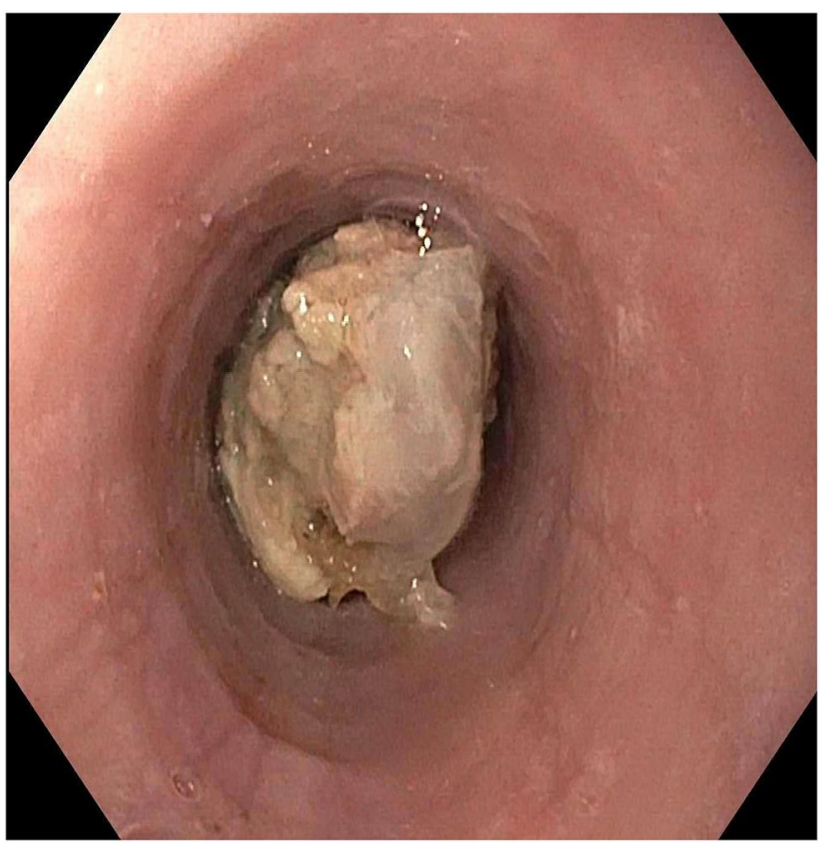

Figure 3 Chicken soft food bolus in oesophagus.

bone $1 / 12$. Blunt objects included tablets still within the foil wrapper $2 / 4$, tree bark $1 / 4$ and rib bone $1 / 4$.

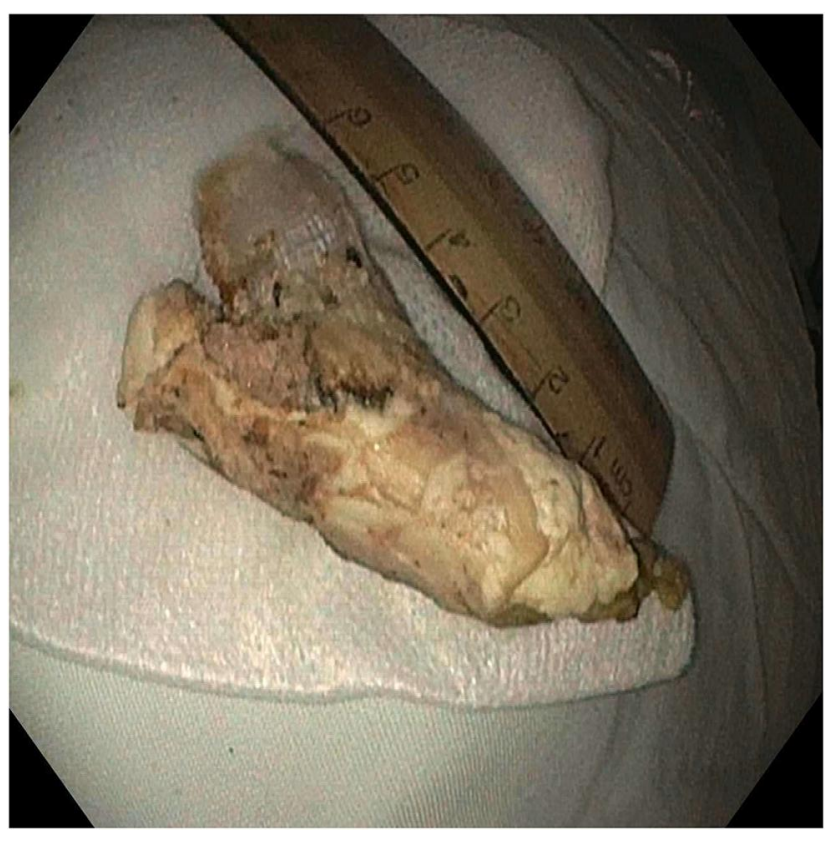

Figure 4 Lamb soft food bolus extracted from oesophagus in one piece.

Time of presentation to hospital was not documented in $5 / 227$. The majority $150 / 222(67.5 \%)$ of patients presented 


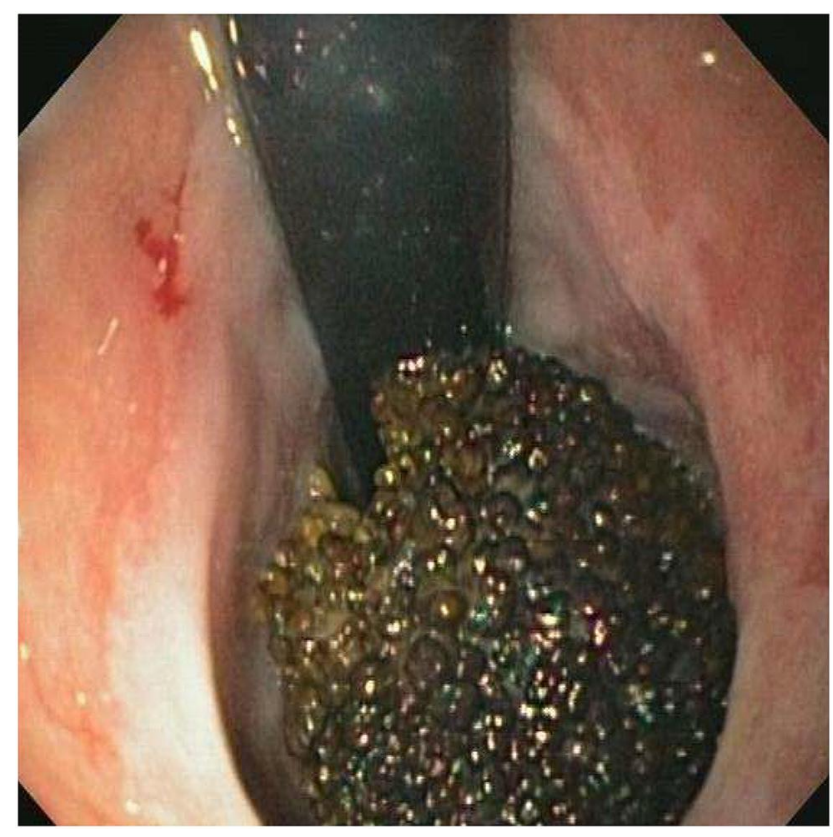

Figure 5 Broccoli floret in the oesophagus of a 64-year-old female patient. The patient had suffered from symptoms of obstruction after eating broccoli nearly 6 days before presenting to hospital.

between 08:00 and 20:00 (daytime) with 72/222 (32.5\%) presenting between 20:00 and 08:00 (overnight).

Signs of complete oesophageal obstruction were documented in 83/227 (37\%) patients at presentation. Concern for oesophageal perforation was documented in 10 patients at presentation of which one underwent a CT scan prior to endoscopy. In total, CT scans were performed in five cases prior to endoscopy, all of whom had ingested foreign bodies (four sharp, one blunt).

Radiographs were performed in 104/204 (50.9\%) of patients presenting with an uncomplicated SFBO. Chest radiographs were performed in 86/204 (42\%), lateral neck radiograph 23/204 (11\%) and abdominal radiograph 7/204 (3\%). Radiographs in multiple planes were performed in 8/ $204(4 \%)$. Of those patients who presented with a SFBO during daytime hours, $50.3 \%$ underwent radiological investigation compared to $52.3 \%$ of patients presenting overnight.

Medical therapies were utilised in an attempt to relieve oesophageal obstructions in the emergency department in $94 / 227(41.4 \%)$ of cases prior to referral to the general medical or endoscopy service. Multiple agents were trialled in 51/94 (54.3\%) of these cases with one agent used in 43/94 (45.7\%), two agents 35/94 (37.2\%), three agents 13/94 (20.2\%), four agents $1 / 94(4.3 \%)$ and five agents 2/94 (2.1\%) of cases. Medical therapies included glucagon 56/94, effervescent drinks 46/94, glyceryl trinitrate spray $32 / 94$, hyoscine butylbromide $22 / 94$, metoclopramide 5/94, diazepam 3/94 and domperidone 1/94. Of those patients who presented overnight, $48.6 \%$ received medical therapies compared to $38.7 \%$ of those presenting during daytime hours.

The time delays between each key time point in the patient's journey from onset of oesophageal obstruction to therapeutic endoscopy are shown below (Table 2). The timings for each individual DHB, daytime versus overnight presentation, type of object causing obstruction (battery, sharp foreign body and SFBO) and presence of complete oesophageal obstruction are also shown.

Overall, the median time from oesophageal obstruction to therapeutic endoscopy was 19 hours. The greatest contributor to this time was the patient-related delay in presentation to hospital (median 7 hours), followed by door to endoscopy time (median 5:12 hours). The median time delay to hospital presentation was markedly shorter during the overnight period (4 hours) than during the daytime period (16:30 hours). However, the median door to endoscopy time was six hours longer for those patients presenting overnight, 10:23 (1:13-22:12) hours, compared to those presenting during daytime hours 4:23 (0:23-11:37) hours. The median time from onset of obstruction to therapeutic endoscopy was found to be shorter in those patients presenting overnight, 16:30 (2:30-66:30) hours than those presenting during daytime hours, median 21:30 (2-258:30) hours.

The median times from onset of obstruction to therapeutic endoscopy all exceeded the guideline standard of removal within 6 hours: Complete oesophageal obstruction (17:45 hours), ingested sharp objects (13:15 hours) or batteries in the oesophagus (18:00 hours). In cases of ingested batteries (8:36 hours) and sharp objects (9:01 hours) the door to endoscopy time was the greatest contributor to time delay. Two patients presented with impacted batteries. The median delay from obstruction to presentation was 5:45 hours with median delay presentation to referral 1:51 (0:42-3:01) hours. Pre-endoscopic medical therapies were utilised in both patients. In the 12 patients in whom an impacted sharp object was endoscopically confirmed, the median (range) delay to presentation was 4 (0:30-19:30) hours, delay to referral 2:13 (1:02$3: 28$ ) hours and the median overall time from onset of obstruction to therapeutic endoscopy was 20 (14-68) hours. 
Table 2 Time Delays (Hours:Minutes) Between Each Key Point in the Patient Journey

\begin{tabular}{|c|c|c|c|c|}
\hline & $\begin{array}{c}\text { Onset of Obstruction } \\
\text { to Hospital } \\
\text { Presentation } \\
\text { Median Time (Range) }\end{array}$ & $\begin{array}{l}\text { Hospital Presentation to } \\
\text { Referral to Endoscopy } \\
\text { Service } \\
\text { Median Time (Range) }\end{array}$ & $\begin{array}{l}\text { Door to } \\
\text { Endoscopy } \\
\text { Time (DtE) } \\
\text { Median Time } \\
\text { (Range) }\end{array}$ & $\begin{array}{c}\text { Overall Time Delay Oesophageal } \\
\text { Obstruction to Therapeutic } \\
\text { Endoscopy } \\
\text { Median Time (Range) }\end{array}$ \\
\hline \multicolumn{5}{|c|}{ DHB to which patient presented } \\
\hline $\begin{array}{l}\text { WDHB } \\
n=148\end{array}$ & $\begin{array}{c}7: 45 \\
(0: 30-255: 00) \\
n=97\end{array}$ & $\begin{array}{c}2: 28 \\
(0: 23-8: 58) \\
n=50\end{array}$ & $\begin{array}{c}4: 48 \\
(0: 27-22: 37) \\
n=143\end{array}$ & $\begin{array}{c}17: 30 \\
(2: 00-258: 30) \\
n=98\end{array}$ \\
\hline $\begin{array}{l}\text { SDHB } \\
n=79\end{array}$ & $\begin{array}{c}5: 15 \\
(0: 30-73: 30) \\
n=70\end{array}$ & $\begin{array}{c}1: 49 \\
(0: 10-14: 00) \\
n=56\end{array}$ & $\begin{array}{c}6: 05 \\
(1: 18-22: 13) \\
n=75\end{array}$ & $\begin{array}{c}19: 00 \\
(2: 30-91: 00) \\
n=7 \mid\end{array}$ \\
\hline $\begin{array}{l}\text { Overall } \\
n=227\end{array}$ & $\begin{array}{c}7: 00 \\
(0: 30-255: 00) \\
n=167\end{array}$ & $\begin{array}{c}2: 09 \\
(0: 10-14: 00) \\
n=106\end{array}$ & $\begin{array}{c}5: 12 \\
(0: 27-22: 37) \\
n=218\end{array}$ & $\begin{array}{c}19: 00 \\
(2: 00-258: 30) \\
n=169\end{array}$ \\
\hline \multicolumn{5}{|c|}{ Daytime vs overnight presentation } \\
\hline $\begin{array}{l}\text { Daytime } \\
(8: 00-20: 00) \\
n=150\end{array}$ & $\begin{array}{c}16: 30 \\
(0: 30-255: 00) \\
n=108\end{array}$ & $\begin{array}{c}1: 52 \\
(0: 16-8: 58) \\
n=55\end{array}$ & $\begin{array}{c}4: 23 \\
(0: 23-11: 37) \\
n=147\end{array}$ & $\begin{array}{c}21: 30 \\
(2: 00-258: 30) \\
n=108\end{array}$ \\
\hline $\begin{array}{l}\text { Overnight } \\
(20: 00-8: 00) \\
n=72\end{array}$ & $\begin{array}{c}4: 00 \\
(0: 30-37: 30) \\
n=59\end{array}$ & $\begin{array}{c}2: 58 \\
(0: 10-14: 00) \\
n=33\end{array}$ & $\begin{array}{c}10: 23 \\
(1: 13-22: 12) \\
n=70\end{array}$ & $\begin{array}{c}16: 30 \\
(2: 30-66: 30) \\
n=58\end{array}$ \\
\hline \multicolumn{5}{|c|}{ Type of impacted object and grade of obstruction } \\
\hline $\begin{array}{l}\text { Signs of complete } \\
\text { oesophageal } \\
\text { obstruction } \\
n=83\end{array}$ & $\begin{array}{c}7: 00 \\
(0: 30-43: 00) \\
n=73\end{array}$ & $\begin{array}{c}\mathrm{I}: 44 \\
(0: 10-8: 40) \\
n=44\end{array}$ & $\begin{array}{c}4: 41 \\
(0: 42-22: 12) \\
n=82\end{array}$ & $\begin{array}{c}17: 45 \\
(2: 00-46: 00) \\
n=72\end{array}$ \\
\hline $\begin{array}{l}\text { Impacted batteries } \\
\mathrm{n}=2\end{array}$ & $\begin{array}{c}5: 45 \\
(3: 00-8: 30) \\
n=2\end{array}$ & $\begin{array}{c}I: 5 I \\
(0: 42-3: 01) \\
n=2\end{array}$ & $\begin{array}{c}8: 36 \\
(2: 50-14: 14) \\
n=2\end{array}$ & $\begin{array}{c}13: 15 \\
(11: 30-15: 00) \\
n=2\end{array}$ \\
\hline $\begin{array}{l}\text { Presumed impacted } \\
\text { sharp objects } \\
n=17\end{array}$ & $\begin{array}{c}5: 00 \\
(0: 30-40.5) \\
n=13\end{array}$ & $\begin{array}{c}\mathrm{I}: 58 \\
(0: 42-3: 28) \\
n=10\end{array}$ & $\begin{array}{c}9: 01 \\
(1: 57-19: 35) \\
n=16\end{array}$ & $\begin{array}{c}18: 00 \\
(11: 30-68: 00) \\
n=13\end{array}$ \\
\hline $\begin{array}{l}\text { SFBO } \\
n=204\end{array}$ & $\begin{array}{c}7: 30 \\
(0: 30-255: 00) \\
n=149\end{array}$ & $\begin{array}{c}2: 07 \\
(0: 10-14: 00) \\
n=88\end{array}$ & $\begin{array}{c}5: 05 \\
(0: 27-22: 37) \\
n=196\end{array}$ & $\begin{array}{c}19: 00 \\
(2: 00-258: 30) \\
n=15 \mid\end{array}$ \\
\hline
\end{tabular}

Note: Time delays are shown for overall obstructions (foreign body and soft food bolus), by DHB, presentations during daytime and overnight, impacted batteries, presumed impacted sharp objects and soft food bolus.

Abbreviation: $n$, number of complete data points.

Following presentation to hospital, the delay to referral to the endoscopy service ranged from 10 minutes to 14 hours. Of the 204 cases who presented with SFBO, the key time points to allow statistical analysis were documented in 88 cases. The median delay from presentation to hospital to referral to the endoscopy service was 127.5 minutes $(95 \%$ CI 96,193). Three patients presenting with a soft food bolus obstruction failed to 
undergo therapeutic endoscopy due to the delay in referral to the endoscopy service, probability 0.034 (95\% CI 0.012, 0.095).

Areas of pressure necrosis within the oesophageal mucosa, directly adjacent to the food bolus, were reported as early as 11 hours after onset of obstruction. Mucosal tears or lacerations were identified in 10 cases, 2 of these cases were in association with impacted sharp foreign bodies and eight cases with an SFBO. In cases where mucosal tears were seen in association with SFBO the median time since onset of impaction was 25 (3-258:30) hours. Two tears required intervention with endoscopically placed clips. In one case, a small oesophageal perforation was conservatively managed.

Two patients died following complications secondary to oesophageal obstruction. Therapeutic endoscopy failed to remove the impacted foreign body (dissembled watch) in one case. This patient proceeded to theatre for open retrieval which was complicated by oesophageal perforation. An Ivor-Lewis oesophagectomy was performed. The patient passed away 3 days after surgery due to postoperative complications. In the second case an 86-yearold patient, presenting with an SFBO, passed away after developing an aspiration pneumonia post endoscopy.
Oesophageal biopsies were performed in 97/204 $(47.5 \%)$ cases at the time of therapeutic OGDs for SFBO. A diagnosis of eosinophilic oesophagitis was made in 32 cases, reflux oesophagitis 29 cases, Schatzki ring 7 cases, benign oesophageal ulceration 4 cases and oesophageal cancer 1. No histopathological abnormality was identified in 24 cases. When combining these figures and prior known risk factors $60.7 \%$ (124/ 204) of cases presenting with SFBO had documented evidence of histopathological oesophageal examination with $80.6 \%$ having an underlying abnormality identified (Figure 6).

\section{Discussion}

While no studies exist to quantify the incidence of oesophageal obstruction in New Zealand, a previous American study estimated an incidence of 13 per 100,000 population of oesophageal food bolus obstruction. ${ }^{4}$ The majority of ingested foreign bodies are accidental events in children ${ }^{1}$ with items such as toys, batteries, coins and magnets most commonly swallowed. ${ }^{5}$ Accidental ingestion of foreign bodies do occur in adults - fish and animal bones being the most common. ${ }^{1}$ Risk factors for accidental ingestion include intoxication and cognitive impairment ${ }^{5}$; however,

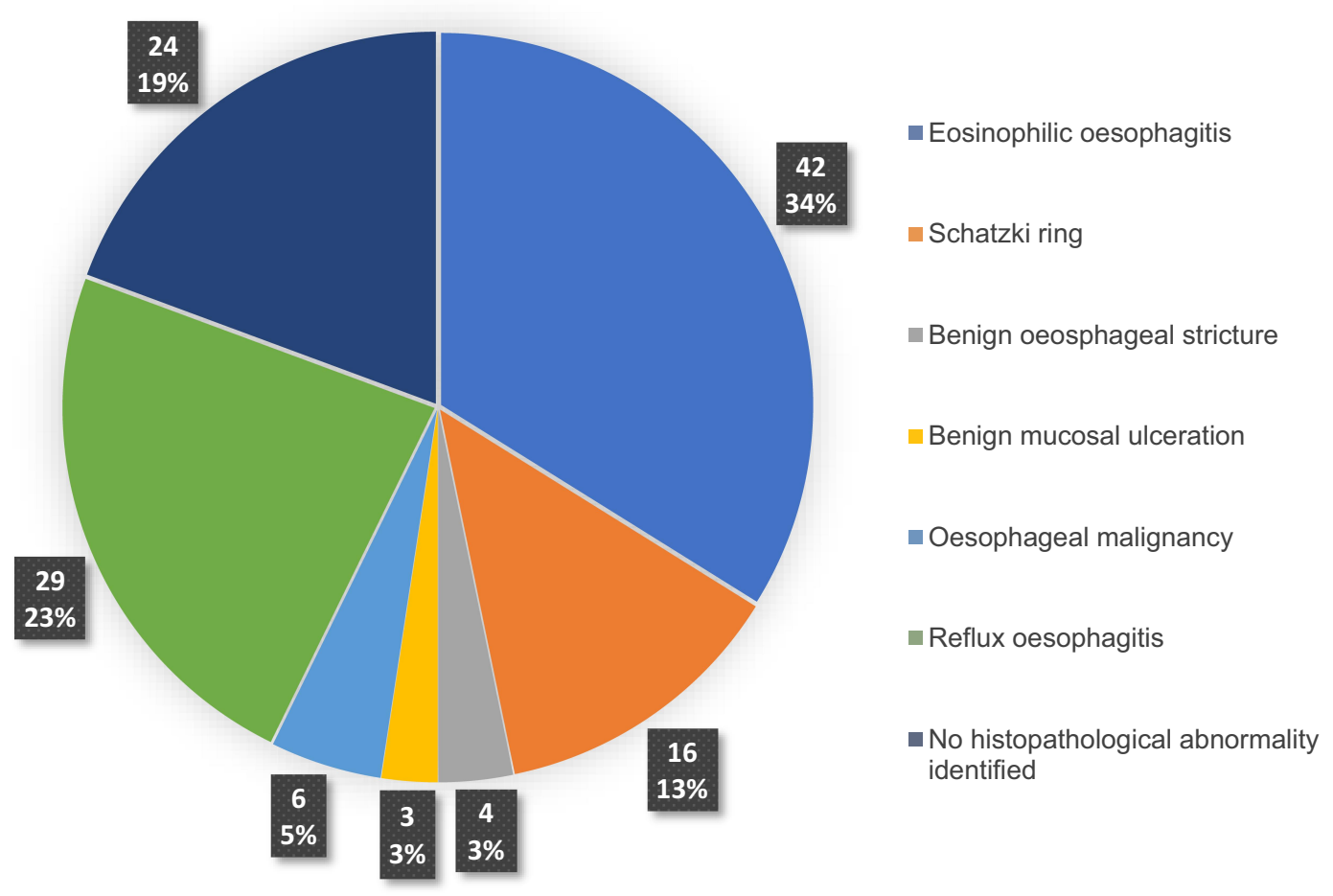

Figure 6 Underlying oesophageal pathologies in those patients presenting with acute oesophageal obstruction (I24/204 cases). 
deliberate ingestions do occur particularly in patients suffering from psychiatric disorders and in prisoners. ${ }^{1,5}$

Impaction of a food bolus or foreign body commonly occurs in the upper third of the oesophagus due to anatomical (cricopharyngeus, aortic arch) and physiological reasons (low-pressure zone at the transition point between striated and smooth muscle fibres). ${ }^{5}$ Patients typically present with acute onset dysphagia, odynophagia, retrosternal pain, hypersalivation, inability to swallow their own saliva, retching and with a sensation of a foreign body stuck in the throat or chest. ${ }^{5}$ Stridor and dyspnoea may be present in patients with airway compromise either secondary to aspiration of saliva or compression of the trachea secondary to the oesophageal obstruction. ${ }^{5}$ The sensation of a retained foreign body and dysphagia can last for several hours after a foreign body or large food bolus has cleared the oesophagus. Hypersalivation and an inability to swallow saliva should raise suspicion for complete oesophageal obstruction. ${ }^{1}$

Acute oesophageal obstruction should be managed in a timely and appropriate manner. ${ }^{1,2}$ A focused history should include the time of symptom onset, type of food or foreign body ingested, any relevant co-morbidities and history of oesophageal pathology. Examination should assess for signs of airway compromise, fever, tachycardia, subcutaneous emphysema or swelling of the neck which may indicate oesophageal perforation. A more general examination should assess fitness for sedation or anaesthesia.

In uncomplicated SFBO, radiological investigations are not recommended due to a false-negative rate of up to $87 \% .{ }^{1}$ Plain radiographs of the neck, chest and abdomen are recommended as first-line management to assess the presence, location, size, configuration of ingested radiopaque foreign bodies, ${ }^{1}$ but the false-negative rate remains high at up to $47 \%{ }^{6}$ Biplanar radiographs are advised if objects are not identified on initial imaging. ${ }^{1}$ Thin metal, wood, plastic, glass, fish or chicken bones are seen particularly poorly on plain radiographs. ${ }^{1,5}$ The sensitivity (90-100\%) and specificity $(93.7-100 \%)$ of CT is superior to the $32 \%$ sensitivity of plain radiographs ${ }^{7}$ for the identification of ingested fish bones. ${ }^{1,5}$

This study identified that radiological investigations are commonly used during the pre-endoscopic assessment of patients presenting with an uncomplicated soft food bolus obstruction. These investigations are not supported by international guidelines, expose patients to unnecessary doses of radiation, incur a financial cost to the health service and could contribute to delays in referral to the endoscopy service. Where clinical concern exists for oesophageal perforation, a CT scan is mandatory. ${ }^{1}$ While 10 patients in this study had documented concerns for perforation only 1 patient underwent a pre-endoscopic CT scan. These patients presented with either tachycardia or fevers which may have been explained by another pathology. None of these patients demonstrated swelling of the neck or subcutaneous crepitus which may be considered more specific clinical sign of perforation.

The 2011 American Society of Gastrointestinal Endoscopy (ASGE) and 2016 European Society Gastrointestinal Endoscopy (ESGE) guidelines recommend emergent endoscopy in the management of acute oesophageal obstruction. ${ }^{1,2}$ While acknowledging a lack of evidence, these guidelines do allow for pre-endoscopic medical therapies such as glucagon or butylscopolamine (Buscopan) to attempt clearance of soft food bolus obstructions if they do not delay endoscopy. However, subsequent to the publication of these guidelines, further studies and review articles have been published which do not support the use of these medical therapies. ${ }^{8,9}$

While this study was not designed to evaluate the efficacy of medical therapies, it has highlighted the frequency of their use in the emergency department. Over $40 \%$ of patients were given medical therapies with over $50 \%$ of these receiving multiple agents. This may be explained by a historic culture of the use of medical therapies in order to try and manage oesophageal obstructions at peripheral hospitals which do not have an endoscopy service on site. Both DHB's operate a "Hub and Spoke" model, eg, at SDHB endoscopy services are only available at Dunedin and Invercargill Hospitals with the peripheral hospitals of Lakes, Dunstan, Gore and Maniototo having to transfer patients requiring therapeutic endoscopy to the "hub" hospitals.

Effervescent drinks are hypothesised to disimpact a food bolus inferiorly by increasing the intraluminal pressure between the bolus and cricopharyngeus above. They are commonly used, especially in resource poor countries, but the evidence supporting their use is limited to case reports and case series. ${ }^{10}$ While a small 2019 South African series documented resolution of complete oesophageal obstruction in $13 / 22(59 \%)$ cases with no adverse events, ${ }^{11}$ other studies have reported complications including aspiration and oesophageal tear. ${ }^{10,12,13}$ A 2005 review article reported success rate of $80 \%$ but a complication rate of $3 \%$ which exceeds that for therapeutic endoscopy. ${ }^{13}$ 
Glucagon is a single-chain polypeptide hormone, involved in glucose homeostasis, which also causes relaxation of the lower oesophageal sphincter and distal oesophagus. While commonly used for acute oesophageal food bolus obstruction it is also supported by low-level evidence. One multicentre prospective, double-blind randomised control trial reported a $37.5 \%$ response to glucagon compared to $31.3 \%$ with placebo. ${ }^{14}$ These results were in keeping with other studies which suggest $30 \%$ of obstructions clear spontaneously. A 2019 systematic review and meta-analysis ${ }^{8}$ supported previous studies in concluding there was no difference in treatment success with glucagon compared to controls, but there was a higher rate of adverse events. ${ }^{9,15}$ Overall treatment was successful in $213 / 706(30.2 \%)$ patients managed with glucagon compared to $158 / 479$ (33\%) patients in the control group. Adverse events including retching, vomiting, burning sensations, hiccups and chest pain were experienced by $15 \%$ in the glucagon group compared to $0 \%$ in the placebo group. ${ }^{8}$ Glucagon has also been demonstrated to be ineffective in the management of oesophageal coin impactions in children. ${ }^{16}$

Hyoscine butylbromide is an anticholinergic agent which inhibits the activation of smooth muscle by parasympathetic pathways inducing relaxation and dilatation. Its use is alleged to originate from 1997 textbook $^{17}$ which misquotes ${ }^{18}$ a 1991 study of 16 patients ${ }^{19}$ in which none of the "spasmolytics" used included hyoscine butylbromide. A literature review does not support its use with no difference in rates of obstruction disimpaction between patients in whom it was used and those who received no medical therapy. ${ }^{19-23}$

Benzodiazepines are hypothesised to relax skeletal muscle found in the proximal oesophagus, and lower oesophageal pressures, by reducing neuron excitability through their effect on the GABA receptor. ${ }^{18}$ There is limited evidence to support their use in oesophageal SFBO with no significant difference in disimpaction rates in patients who received diazepam/glucagon combination compared to placebo. ${ }^{14}$

A literature search for studies or case reports investigating the use GTN, metoclopramide or domperidone as medical therapies for the management of acute oesophageal obstruction yielded no results.

Therapeutic endoscopy is recommended as first-line management for acute oesophageal obstruction. ${ }^{1}$ It is sensitive, safe, effective and allows for the identification of any predisposing pathological process. Arranging a therapeutic endoscopy can take time to co-ordinate, requiring an endoscopist and support staff on site, as well as an appropriate environment in which to perform the procedure. Where patients have a potentially compromised airway anaesthetic and theatre support may be required. To try and minimise the time to therapeutic endoscopy patients presenting with acute oesophageal obstruction should therefore be identified, triaged and referred to the endoscopy services without delay.

A patient's journey from presentation to hospital to discharge is complex and may be affected by many variables of which this retrospective study was not designed to the assess (Figure 1). We identified that only $5 \%$ of patients $(6 / 106)$ were referred to the endoscopy services promptly (within 30 minutes of arrival) with $95 \%$ of patients waiting between 30 minutes and 14 hours. More than half underwent multiple unnecessary investigations and more than $40 \%$ were administered medical therapies which lack therapeutic evidence. While our statistical analysis suggests that the probability of failing to undergo therapeutic endoscopy for a soft food bolus obstruction within the recommended timeframes due to the delay in referral to the endoscopy service was low $(0.034,95 \% \mathrm{CI}$ $0.012,0.095)$ reducing delays in the patient's journey is clearly in their best interest. Factors affecting management at presentation may include busyness of the emergency department, seniority/experience/knowledge base of emergency department staff, availability and practicality of local management pathways and a need to transfer to another hospital with endoscopy services.

While this retrospective study cannot identify every factor contributing to longer overnight delays, we identified that patients presenting overnight with oesophageal obstruction waited 6 hours longer to undergo therapeutic endoscopy compared to those patients presenting during the daytime. Patients presenting overnight were given more medical therapies (48.6\% vs $38.7 \%)$ which may indicate difficulties in accessing endoscopy services overnight.

Guidelines advise all IFB and SFBO should be removed within 24 hours as the risk of major complications (perforation \pm mediastinitis, retropharyngeal abscess and aorto-oesophageal fistula) increases 14.1 times beyond this timeframe. ${ }^{1,24}$ A retrospective cohort study also reported a lower incidence of odynophagia and oesophageal ulcers when disimpaction was performed within 24 hours. ${ }^{25}$ 
Where clinical signs suggest complete oesophageal obstruction secondary to SFBO, guidelines suggest therapeutic endoscopy should be performed within a maximum 6 hours. ${ }^{1}$ Sharp-pointed foreign bodies, button batteries and magnets should also be removed within this expedited time frame. The rate of perforation for sharp-pointed foreign bodies is reported up to $35 \%$, while button batteries are high risk of causing perforation through pressure necrosis and electrical discharge. ${ }^{1}$ We identified complications secondary to prolonged oesophageal obstructions, with pressure necrosis reported as early as 11 hours after onset of obstruction. The estimated rate of oesophageal laceration during therapeutic OGD for oesophageal obstruction is $0.7 \% .{ }^{18}$ In this study, mucosal tears were seen in 8/204 (3.9\%) of SFBO and 2/12 (16.6\%) of impacted sharp foreign objects.

Surgical intervention is estimated to be required in $1-3 \%$ of the patients with acute oesophageal obstruction due to complications such as perforation, irretrievable foreign bodies, mediastinitis, pleural empyema, fistula, and bleeding. ${ }^{1,5}$ Endoscopists should take these potential risks of prolonged impaction into account when formulating decision-making about whether to perform therapeutic endoscopy for oesophageal impaction in or out of hours. Further, hospital systems need to ensure that staff performing endoscopy out of hours are able to do so safely, including safe roster and rest periods.

While a number of outcome measures may be utilised to analyse differences between therapeutic endoscopy delivered on time compared to delayed, these outcome measures/complications are rare and this retrospective study was not designed or powered to investigate this. In addition while objective outcome measures such as oesophageal perforation, duration of procedure and failure of therapeutic endoscopy may be utilised, they do not assess important subjective patient outcomes such as pre- and post-therapeutic endoscopy pain scores. These are important outcome measures to consider when attempting to improve delivery of patient centred care and when updating guidelines for this presentation.

Up to $75 \%$ of the patients suffering from acute oesophageal obstructions are estimated to have underlying oesophageal pathology; therefore, diagnostic work-up for a cause is recommended. ${ }^{1}$ Our study identified $80.6 \%$ of the patients had an underlying oesophageal abnormality with eosinophilic oesophagitis, and reflux oesophagitis being the most common abnormality. A lack of appropriate investigation and follow-up for an underlying pathology is a predictor of recurrent impactions, ${ }^{6}$ in our study $36.4 \%$ had not undergone histopathological assessment. In this study, the most common risk factor was a previous oesophageal obstruction, 65/227 (28.6\%). Patients who present with oesophageal obstruction should be educated at discharge about any underlying pathology and regarding what to do should they experience another obstruction so as to reduce patient-related delay to presentation.

In order to minimise delays, reduce unnecessary exposure to radiological investigations, avoid medical therapies and expedite therapeutic endoscopy. Waitemata DHB developed a clinical guideline to guide emergency and acute care teams in managing patients presenting with acute oesophageal obstructions secondary to soft food boluses and foreign bodies (Appendix 2). This pathway is also freely available in the New Zealand Society of Gastroenterology website.

\section{Acknowledgments}

We would like to thank Dr Marius Van Rijnsoever, Consultant Gastroenterologist, Waitemata DHB for his assistance with Provation $^{\circledR}$ data searches and Robin Willink, Senior Research Fellow and Biostatistician at Otago University for his guidance and assistance using R Statistical Software.

\section{Funding}

There are no external sources of funding to declare.

\section{Disclosure}

The authors have no conflicts of interest to declare.

\section{References}

1. Birk M, Bauerfeind P, Deprez P, et al. Removal of foreign bodies in the upper gastrointestinal tract in adults: European Society of Gastrointestinal Endoscopy (ESGE) clinical guideline. Endoscopy. 2016;48(05):489-496. doi:10.1055/s-0042-100456

2. Ikenberry S, Jue TL, Anderson MA, et al. Management of ingested foreign bodies and food impactions. Gastrointest Endosc. 2011;73 (6):1085-1091. doi:10.1016/j.gie.2010.11.010

3. New Zealand Society of Gastroenterology Oesophageal Obstruction Pathway; 2019. Available from: https://nzsg.org.nz/resources/. Accessed May 14, 2021.

4. Longstreth GF, Longstreth KJ, Yao JF. Esophageal food impaction: epidemiology and therapy. A retrospective, observational study. Gastrointest Endosc. 2001;53(2):193-198. doi:10.1067/mge.2001. 112709

5. Chirica M, Kelly MD, Siboni S, et al. Esophageal emergencies: WSES guidelines. World J Emerg Surg. 2019;14(1):26. doi:10.1186/ s13017-019-0245-2

6. Pfau PR. Removal and management of esophageal foreign bodies. Tech Gastrointest Endosc. 2014;16(1):32-39. doi:10.1016/j.tgie.20 13.10.004 
7. Ngan JH, Fok PJ, Lai EC, Branicki FJ, Wong J. A prospective study on fish bone ingestion. Experience of 358 patients. Ann Surg. 1990;211(4):459-462. doi:10.1097/00000658-199004000-00012

8. Peksa GD, DeMott JM, Slocum GW, Burkins J, Gottlieb M. Glucagon for relief of acute esophageal foreign bodies and food impactions: a systematic review and meta-analysis. Pharmacother $J$ Hum Pharmacol Drug Ther. 2019;39(4):463-472. doi:10.1002/phar.2236

9. Lorrains J. BET 1: use of glucagon for oesophageal food bolus impaction: table 1. Emerg Med J. 2015;32(1):85.2-88. doi:10.1136/ emermed-2014-204467.1

10. Mohammed SH, Hegedüs V. Dislodgement of impacted oesophageal foreign bodies with carbonated beverages. Clin Radiol. 1986;37 (6):589-592. doi:10.1016/S0009-9260(86)80034-4

11. Baerends EP, Boeije T, Van Capelle A, Mullaart-Jansen NE, Burg MD, Bredenoord AJ. Cola therapy for oesophageal food bolus impactions a case series. Afr J Emerg Med. 2019;9(1):41-44. doi:10.1016/j.afjem.2018.09.005

12. Zimmers TE, Chan SB, Kouchoukos PL, Mirande H, Noy Y, VanLeuven B. Use of gas-forming agents in esophageal food impactions. Ann Emerg Med. 1988;17(7):693-695. doi:10.1016/ S0196-0644(88)80613-9

13. Lee J, Anderson RS. Effervescent agents for oesophageal food bolus impaction. Emerg Med J. 2005;22(2):123-124. doi:10.1136/emj.20 04.022053

14. Tibbling L, Bjorkhoel A, Jansson E, Stenkvist M. Effect of spasmolytic drugs on esophageal foreign bodies. Dysphagia. 1995;10 (2):126-127. doi:10.1007/BF00440084

15. Robbins MI, Shortsleeve MJ. Treatment of acute esophageal food impaction with glucagon, an effervescent agent, and water. $\mathrm{Am}$ J Roentgenol. 1994;162(2):325-328. doi:10.2214/ajr.162.2.8310919

16. Mehta DI, Attia MW, Quintana EC, Cronan KM. Glucagon use for esophageal coin dislodgment in children: a prospective, double-blind, placebo-controlled trial. Acad Emerg Med. 2001;8(2):200-203. doi:10.1111/j.1553-2712.2001.tb01291.x
17. Birchall MA, Croft CB. Examination and endoscopy of the upper aerodigestive tract. In: Kerr AG, editor. Scott-Brown's Otolaryngology: Laryngology. 6th. Vol. 5. Oxford: Butterworth Heinemann; 1997.

18. Leopard D, Fishpool S, Winter S. The management of oesophageal soft food bolus obstruction: a systematic review. Ann $R$ Coll Surg Engl. 2011;93(6):441-444. doi:10.1308/003588411X588090

19. Tibbling L, Stenquist M. Foreign bodies in the esophagus. A study of causative factors. Dysphagia. 1991;6(4):224-227. doi:10.1007/ BF02493532

20. Anderson R, Lee J. Buscopan for oesophageal food bolus impaction. Emerg Med J. 2007;24(5):360-361. doi:10.1136/emj.2007.048496

21. Basavaraj S, Reddy CE, Dempster J, Reddy K, Kang M. Buscopan (HYOSCINE BUTYLBROMIDE) in the management of food bolus obstruction in oesophagus: randomised controlled trial. Eur Arch Otorhinolaryngol. 2005

22. Basavaraj S, Penumetcha KR, Cable HR, Umapathy N. Buscopan in oesophageal food bolus: is it really effective? Eur Arch Otorhinolaryngol. 2005;262(7):524-527. doi:10.1007/s00405-0040852-7

23. Thomas L, Webb C, Duvvi S, Jones T, Reddy KTV. Is buscopan effective in meat bolus obstruction? Is buscopan effective in meat bolus obstruction? Clin Otolaryngol. 2005;30(2):183-185. doi:10.1111/j.1365-2273.2004.00931.x

24. Seng Loh K, Siang Tan LK, Smith JD, Hian Yeoh K, Dong F. Complications of foreign bodies in the esophagus. Otolaryngol Neck Surg. 2000;123(5):613-616. doi:10.1067/mhn.2000.110616

25. Wu WT, Chiu CT, Kuo CJ, et al. Endoscopic management of suspected esophageal foreign body in adults. Dis Esophagus. 2011;24 (3):131-137. doi:10.1111/j.1442-2050.2010.01116.x

\section{Publish your work in this journal}

Clinical and Experimental Gastroenterology is an international, peerreviewed, open access, online journal publishing original research, reports, editorials, reviews and commentaries on all aspects of gastroenterology in the clinic and laboratory. This journal is indexed on American Chemical Society's Chemical Abstracts Service (CAS)
The manuscript management system is completely online and includes a very quick and fair peer-review system, which is all easy to use. Visit http://www.dovepress.com/testimonials.php to read real quotes from published authors. 